\title{
Intorno alle possibili distribuzioni della massa nell'interno della Terra.
}

\author{
(Di P. PIKETTI, a Pisa.)
}

La forma di una superficie di livello esteriore alla massa terrestre può, al dì d'oggi, ritenersi abbastanza ben conosciuta nel suo complesso. Le ben note proprietà delle funzioni armoniche provano che la conoscenza di una tale superficie, e, insieme, della massa totale della Terra e della velocità angolare del movimento diurno, sono sufficienti a determinare in modo nou ambiguo la grandezza e la direzione della accelerazione di gravità in ogni punto esterno al contorno $(*)$, solido o liquido, della massa terrestre.

Una tale determinazione è pertanto indipendente da speciali ipotesi sul modo di variare della densità nell'interno del globo; epperò da tali arbitrarie ipotesi risulta pure indipendente il contributo che le misurazioni della gravità apportano allo studio del Geoide. Questa verità è stata per la prima rolta enunciata da Stokes nel 1849 e laverla posta in evidenza costituisce senza dubbio il più grande progresso che, dal punto di vista del problema fisico, la teoria della figura della Terra abbia fatto dai tempi di Clamnaut fino ai dì nostri.

Ma si comprende come, reciprocamente, la conoscenza della figura del Geoide (e quindi la determinazione teorica della espressione analitica della funzione potenziale dell'attrazione terrestre al di fuori della Terra) ben poche notizie ci possa porgere $\left({ }^{* *}\right)$ sul modo di distribuzione della densità nell'interno. Infiniti modi differenti di rariazione della densità entro uno spazio

$\left.{ }^{*}\right)$ Si dovrebbe comprendere, teoricamente, nella massa terrestre, anche l'atmosfera, ma si dimostra facilmente che, nello studio delle superficie di livello terrestre, l'influenza di questa è del tutto trascurabile.

(**) Quando, ben inteso, si escludano le considerazioni idrostatiche che derivano dalla ipotesi dello stato primitivamente fluido. 
chiuso corrispondono ad una stessa espressione della funzione potenziale attrattiva all'esterno. Pur tuttavia questi differenti modi hanno talune qualità in comune, sicchè le ipotesi sulla interna distribuzione della massa terrestre restano legate, in causa della conoscenza della superficie esterna, a certe condizioni. Porre in evidenza quelle qualità comuni e queste condizioni è lo scopo del presente lavoro $\left(^{*}\right)$.

Due sono i problemi che qui si presentano: Trovare qualcuna (una almeno) distribuzione di massa che sia compatibile colla forma osservata del Geoide; e vedere poi in quali modi si possa, nell'interno, immaginare alterata la distribuzione senza che resti alterata la attrazione Newtoniana all'esterno, nè, quindi, la forma delle superficie di livello esteriori. Cominceremo dallo studio di questo secondo problema.

(*) Per quanto io mi sappia, i problemi che formano oggetto di questo lavoro sono stati pochissimo trattati. Tipi classici di sistemi equivalenti rispetto alla attrazione esterna sono i notissimi strati di livello di CHASLEs; ma questi tipi hanno poca importanza dal punto di vista del problema da me trattato, in quanto involgono la esistenza di densití superficiali finite che io naturalmente debbo escludere. Un cenno appena della quistione sulla « internal distribution of Matter which shall produce a given potential at the surface of a gravitating Mass» si ha in una breve Nota di G. G. Stokfs portante appunto il titolo: On the internal distribution, etc. (1867) (Mathem. and Phys. Papers. Vol. 4, pag. 277).

Un modo mol to ingegnoso di costruire sistemi equivalenti rispetto alla attrazione esterna è stato indicato da G. V. Schtaparelut nelle Jezioni di Meccanica Celeste da lui' date nella Università di Pavia nell'anno scolastico 1875.76. Egli osserva come si possa sostituire ad una massa concentrata in un punto $P$ una massa, di eguale grandezza totale, distribuita per strati sferici omogenei di centro $P$, senza alterare la attrazione esterna. Con questo procedimento (che si può ripetere a piacere e che Egli chiama: espansione sferica) si possono sostituire ad un corpo infiniti altri di eguale attrazione esterna e in particolare costruire corpi i quali attraggono all'esterno come se tutta la loro massa fosse concentrata in un punto. Lo Schialarklu dimostra che questo punto è il centro di massa e che gli assi principali d'jnerzia di un tal corpo sono fra loro eguali. Questi due teoremi sono caso particolare di quelli contenuti nel $\$ 5$ del presente lavoro. La costruzione da me studiata nei $\$ \$ 12,13$ corrisponde in certo modo all'idea della espansione sferica. Alla memoria del grande Astronomo, che ebbe la bontà di mandarmi, per lettera, notizie e citazioni bibliografiche mando un riverente saluto.

Delle idee contenute nelle lezioni di Schiaparentu ebbi notizia quando le idee svolte in questo mio lavoro erano da un pezzo stabilite. Purtroppo quelle lezioni sono pochissimo conosciute, essendo state soltanto raccolte solto forma di appunti da pochi eletti scolari; ad uno dei quali, il mio carissimo collega prof. G. A. Macigi, debbo la conoscenza delle dette lezioni e il gentile imprestito di quegli appunti. 
Gorpl di attraztone nUlla e corpi di EgUale atTtrazjone.

\$ 1. Enunciato del problema. I punti principali che riguardano questo secondo problema furono da me sommariamente esposti in una Nota pubblicata l'anno scorso nei Rendiconti della R. Accademia dei Lincei (*). Qui intendo svolgerli più ampiamente.

Se in uno spazio finito $\tau$ a tre dimensioni, limitato da una superficie chiusa $S$, si immagina distribuita una massa con densità spaziale finita, e se, intorno al valore della densità nel punto generico $M(x, y, z)$, si fanno due differenti ipotesi esprimendola successivamente con

$$
k=k(x, y, z), \quad k^{\prime}=k^{\prime}(x, y, z)
$$

(ove i secondi membri sono funzioni finite delle coordinate), la condizione necessaria e sufficiente perche, per ogni punto esterno alla superficie $S\left({ }^{* *}\right)$ risultino uguali, in grandezza e direzione, le attrazioni esercitate da quelle due differenti distriluuzioni di massa, è che si abbia

$$
\int_{i} \frac{k}{r} d \tau=\int_{i} \frac{k^{\prime}}{r} d \tau
$$

dove $r$ è la distanza dell'elemento gencrico $d \tau$ dello spazio $\tau$ da un punto arbitrario $P\left(x_{1}, y_{1}, z_{1}\right)$ esterno alla $S\left({ }^{* * *}\right)$. Si dirà allora che quelle due distribuzioni, o, come più semplicemente diremo, i due corpi $\dot{C}$ e $C^{\prime}$ sono equivalenti rispetto alla altrazione esterna. Indicando con $h$ la funzione

$$
h=k^{\prime}(x, y, z)-k(x, y, z)
$$

e immaginando che la densità possa assumere valori sia positivi, sia negativi, un corpo tittizio $C^{\prime \prime}$ la cui densità nel punto $M(x, y, z)$ sia $h$ avrà, per

(*) Corpi equivalenti rispetto alla attrazione newtoniana esterna. Rendic. Lincei, XVIII, $1 .^{\circ}$ sem., fasc. $5^{\circ}{ }^{\circ}$

(**) E quinđi anche pei punti della superficie stessa, per la proprietà di continuità della funzione potenziale.

(***) E superfluo osservare che le due funzioni potenziali non possono differire di una costante, perchè debbono entrambe annullarsi nei punti $P$ a distanza infinita. 
la (1), funzione potenziale nulla sopra ogni punto esterno alla $S$ (o della $S$ ). Lo chiameremo corpo di altrazione esterna nulla, relativo allo spazio т. Eे chiaro che la ricerca del come possa alterarsi la distribuzione di massa entro uno spazio - in guisa che non cangi, in grandezza e direzione, la attrazione sopra ogni punto esterno, equivale alla ricerca di tutti i corpi di attrazione nulla relativi allo spazio stesso.

\$ 2. Condizioni alle quali soddisfanno $i$ corpi di altrazione nulla. Il punto $P\left(x_{1}, y_{1}, z_{1}\right)$ può essere supposto abbastanza lontano dall'origine delle coortinate perchè l'inversa della distanza di $P$ da ogni punto $M$ dello spazio $\tau$ possa essere espressa dallo sviluppo

$$
\frac{1}{r}=\sum_{0}^{\infty} \frac{p^{n}}{p_{1}^{n+1}} P_{n}
$$

dove $\rho, \rho_{1}$ sono le distanze dei punti $M, P$ dall'origine $O$, e $P_{n}$ indica il $p o-$ linomio d'ordine $n$ di Legendre, avente per argomento il coseno dell'angolo $M O P$. La condizione (1) equivale alla

$$
\int_{\tau} \frac{h}{r} d \tau=\sum_{0}^{\infty} \frac{1}{\rho_{1}^{n+1}} \int_{i} h \rho^{n} P_{n} \cdot d \tau=0,
$$

e, poichè questa dev'essere verificata qualunque sia $p_{1}$, dovranno essere verificate le relazioni.

$$
\int h p^{n} P_{n} \cdot d \tau=0 \quad(n=0,1,2, \ldots, \infty)
$$

(Sopprimiamo d'or innanzi l'indice $\tau$ presso al segno integrale; intendendo che gl'integrali, salvo contrario avviso, si riferiscano a tutto lo spazio $~$ chiuso dalla superficie $S$ ).

Indichiamo $\operatorname{con} \theta, v$ la colatitudine e la longitudine del punto $M$, con $\theta_{1}, v_{1}$ quella di $P$ in un arbitrario sistema di coordinate polari avente origine in $O$, in guisa che

$$
\cos (M O P)=\cos \theta \cdot \cos \theta_{1}+\operatorname{sen} \theta \cdot \operatorname{sen} \theta_{1} \cdot \cos \left(v-v_{1}\right) .
$$

Introdotta questa espressione di $\cos (M O P)$ nella $P_{n}$, questa può porsi, come è noto, sotto la forma

$$
\begin{gathered}
P_{n}=P_{n}(\mu) \cdot P_{n}(\nu)+ \\
+2 \sum_{i=1}^{n} \frac{\operatorname{sen}^{i} \theta \cdot \operatorname{sen}^{i} \theta_{1}}{(n-i+1) \ldots(n+i)} \frac{d^{i} P_{n}(\mu)}{d \mu^{i}} \frac{d^{i} P_{n}(\nu)}{d \nu^{i}} \cos \left(i \nu-i \nu_{1}\right) .
\end{gathered}
$$


In questa formola $P_{n}(v), P_{a}(v)$ sono ottenuti sostituendo le lettere $u, v$ risp. ${ }^{\circ}$ alla lettera $x$ nel polinomio $X_{n}=P_{n}(x)$ di LEgENDRE; dopo eseguite le operazioni indicate, s'intende alla lettera $\mu$ sostituito $\cos \theta$, alla $\nu \cos \theta_{1}$. Introducendo l'espressione (3) nella (2) ed osservando che la (2) dev'essere verificata per ogni valore di $\theta_{1}$ fra 0 e $\pi$ e di $v_{1}$ fra 0 e $2 \pi$, si vede che la (2) si spezza nelle $2 n+1$ condizioni

$$
\begin{array}{r}
\int h \rho^{\prime \prime} P_{n}(\cos \theta) \cdot d \tau=0 \\
\int h \rho^{n} \frac{d^{i} P_{n}(\mu)}{d \varphi^{i}} \operatorname{sen}^{i} \theta \cdot \cos i v \cdot d \tau=0 \\
\int h \rho^{\prime \prime} \frac{d^{i} P_{n}(\mu)}{d \mu^{i}} \operatorname{sen}^{i} \theta \cdot \operatorname{sen} i v \cdot d \tau=0 .
\end{array}
$$

\$ 3. Caso della distribuzione simmetrica intorno ad un asse. Se la massa è simmetricamente distribuita intorno all'asse $\theta=0$, la $h$ non dipende da $v$, e le (5) sono senz'altro verificate. Le sole condizioni a verificarsi sono allora le (4). Ma è facile vedere che le (4) esprimono le condizioni necessarie e sufficienti perchè sia nulla la f.' potenziale del corpo sopra ogni punto, esterno al corpo, dell'asse $\theta=0$. Resta così provato un noto teorema di Legendne, che: se la f: potenziale di un solido onrogeneo di rotazione (o più generalmente di una massa distribuita simmetricamente intorno ad un asse) è conosciuta per ogni punto esterno dell'asse, essa resta, per questo, del tutto determinata per ogni altro punto esterno.

§ 4. Relazioni fra $i$ momenti d'inerzia di grado qualunque in un corpo di attrazione nulla.

Le (4) (5) conducono facilmente a stabilire delle relazioni fra i momenti d’inerzia del grado $n$. Poniamo, per esempio, $n=4$. Si ha

$$
\begin{aligned}
& P_{4}(\mu)=P_{4}(\cos \theta)=\frac{1}{8}\left(35 \cos ^{4} \theta-30 \cos ^{2} \theta+3\right) \\
& \frac{2}{5} \frac{d P_{4}(\mu)}{d \mu}=7 \cdot \cos ^{3} \theta-3 \cos \theta \\
& \frac{2}{15} \frac{d^{2} P_{4}(\mu)}{d \mu^{2}}=7 \cos ^{3} \theta-1 \\
& \frac{2}{105} \frac{d^{3} P_{4}(\mu)}{d \lambda^{3}}=\cos \theta, \quad \frac{2}{105} \frac{d^{4} P_{4}(\mu)}{d \mu_{4}^{4}}=1 .
\end{aligned}
$$


La (4) dà pertanto per $n=4$

$$
\int h p^{4}\left(35 \cdot \cos ^{4} \theta-30 \cos ^{2} \theta+3\right) d \tau
$$

e le (5) forniscono altre otto condizioni che per brevità raggruppiamo due a due scrivendole così :

$$
\begin{aligned}
& \int h \rho^{4}\left(7 \cos ^{3} \theta-3 \cos \theta\right)(\cos v+\sqrt{-1} \operatorname{sen} v) \operatorname{sen} \theta \cdot d \tau=0 \\
& \int h \rho^{4}\left(7 \cos ^{2} \theta-1\right)(\cos 2 v+\sqrt{-1} \cdot \operatorname{sen} 2 v) \operatorname{sen}^{2} \theta \cdot d \tau=0 \\
& \int h \rho^{4} \cos \theta(\cos 3 v+\sqrt{-1} \operatorname{sen} 3 v) \operatorname{sen}^{3} \theta \cdot d \tau=0 \\
& \int h \rho^{4}(\cos 4 v+\sqrt{-1} \operatorname{sen} 4 v) \operatorname{sen}^{4} \theta \cdot d \tau=0 .
\end{aligned}
$$

Indichiamo, per semplicita, con $\left[a^{r} b^{s} c^{\prime}\right]$ il momento d'inerzia di grado $n=r+s+t$ espresso dall'integrale

$$
\int h \cdot x^{n} y^{s} z^{t} \cdot d \tau=\left[a^{r} b^{s} c^{t}\right]
$$

Passando dalle coordinate cartesiane alle polari, scriveremo

$$
\begin{gathered}
\rho^{2}=x^{2}+y^{2}+z^{2} \\
x=\rho \operatorname{sen} \theta \cos v, \quad y=\rho \operatorname{sen} \theta \operatorname{sen} v, \quad z=\rho \cos \theta \\
\rho^{i} \cdot \operatorname{sen}^{i} \theta(\cos i v+\sqrt{-1} \operatorname{sen} i v)=(x+\sqrt{-1} y)^{i}
\end{gathered}
$$

sicchè le (6) (7) diverranno

$$
\begin{aligned}
& \int h\left(35 \cdot z^{4}-30 \cdot \rho^{2} z^{2}+3 p^{4}\right) \cdot d \tau=0 \\
& \int h\left(7 z^{3}-3 z p^{2}\right)(x+\sqrt{-1} y) \cdot d \tau=0 \\
& \int h\left(7 z^{2}-\rho^{2}\right)(x+\sqrt{-1} y)^{2} \cdot d \tau=0 \\
& \int h z(x+\sqrt{-1} y)^{3} \cdot d \tau=0 \\
& \int h(x+\sqrt{-1} y)^{4} d \tau=0 .
\end{aligned}
$$


Adottando la notazione (8), queste dànno luogo a nove relazioni fra i momenti d'inerzia del quarto grado, che nel modo più spiccio troviamo come segue. Dalle ultime due sì ha

$$
\begin{aligned}
{\left[a^{3} c\right] } & =3\left[a b^{2} c\right], & & {\left[b^{3} c\right]=3\left[a^{2} b c\right] } \\
{\left[a^{4}\right]+\left[b^{4}\right] } & =6\left[a^{2} b^{2}\right], & & {\left[a^{3} b\right]=\left[a b^{3}\right] . }
\end{aligned}
$$

Permutando fra loro le lettere $a, b, c$

$$
\begin{gathered}
{\left[a^{3} b\right]=3\left[a b c^{2}\right]} \\
{\left[b^{4}\right]+\left[c^{4}\right]=6\left[b^{2} c^{2}\right], \quad\left[c^{4}\right]+\left[a^{4}\right]=6\left[a^{2} c^{2}\right]} \\
{\left[a^{3} c\right]=\left[a c^{3}\right], \quad\left[b^{3} c\right]=\left[b c^{3}\right] .}
\end{gathered}
$$

Abbiamo così le nove relazioni cercate, delle quali, com'è facile verificare, quelle che si dedurrebbero dalle prime tre delle (9) sono conseguenza identica.

In generale il numero dei differenli momenti d'inerzia di grado $n$ di un corpo è $\frac{1}{2}(n+1)(n+2)$. La assegnazione della funyione potenziale esterna del corpo lascia adunque arbitrarii, per ogni valore di $n$,

$$
\frac{(n+1)(n+2)}{2}-(2 n+1)=\frac{n(n-1)}{2}
$$

momenti d'inerzia di grado $n$.

$\S 5$. Casi $n=0,1,2$. Particolarmente interessanti sono le conseguenze che dalle condizioni (4) (5) si deducono per i casi $n=0,1$, 2. Si ottiene: per $n=0$ la sola condizione

$$
\int h \cdot d \tau=0
$$

per $n=1$ le tre condizioni

$$
\int h x \cdot d \tau=\int h y \cdot d \tau=\int h z \cdot d \tau=0
$$

per $n=2$, ricordando che $P_{2}(u)=\frac{1}{2}\left(3 \mu^{*}-1\right)$ si hanno le cinque 
condizioni

$$
\begin{gathered}
\int h\left(3 \cos ^{2} \theta-1\right) \rho^{2} \cdot d \tau=0 \\
\int h \rho^{2} \cos \theta \operatorname{sen} \theta \cos v \cdot d \tau=0, \quad \int h \rho^{2} \cos \theta \operatorname{sen} \theta \operatorname{sen} v \cdot d \tau=0 \\
\int h \rho^{2} \operatorname{sen}^{2} \theta\left(\cos ^{2} v-\operatorname{sen}^{2} v\right) \cdot d \tau=0, \quad \int h \rho^{2} \operatorname{sen}^{2} \theta \operatorname{sen} v \cos v \cdot d \tau=0
\end{gathered}
$$

donde senza difficoltà

$$
\begin{gathered}
\int h x^{2} \cdot d \tau=\int h y^{2} \cdot d \tau=\int h z^{2} \cdot d \tau \\
\int h x y \cdot d \tau=0, \quad \int h y z \cdot d \tau=0, \quad \int h z x \cdot d \tau=0 .
\end{gathered}
$$

Se dalla considerazione del corpo di attrazione nulla, passiamo a quella dei due corpi $C, C^{\prime}$ le cui densità generiche sono $k, k^{\prime}$ rispett. $;$ posto nelle (10) (11) (12) (13) $k-k^{\prime}$ in luogo di $h$, abbiamo le seguenti condizioni necessarie affinchè $\mathrm{i}$ due corpi siano equivalenti rispetto alla attrazione esterna

$$
\begin{gathered}
\int k \cdot d \tau=\int k^{\prime} \cdot d \tau \\
\int k x \cdot d \tau=\int k^{\prime} x \cdot d \tau, \quad \text { ed altre due analoghe, } \\
\int k x y \cdot d==\int k^{\prime} x y \cdot d \tau, \\
\left.\int k\left(x^{2}-y^{2}\right) \cdot d \tau=\int k^{\prime}\left(x^{2}-y^{2}\right) \cdot d \tau\right\} \\
\left.\int k\left(x^{2}-z^{2}\right) \cdot d \tau=\int k^{\prime}\left(x^{2}-z^{2}\right) \cdot d \tau\right\}
\end{gathered}
$$

La $\left(10^{\prime}\right)$ esprime che i due corpi debbono avere ugual massa, ciò che era senz'altro evidente per le proprietà limite della f." potenziale nei punti a distanza infinita.

Le $\left(11^{\prime}\right)$ esprimono che se l'origine delle coordinate è assunta nel centro di massa del corpo $C$, in essa deve pur cadere il centro di massa del corpo $C^{\prime}$. Vale a dire : 
a) $i$ due corpi hanno lo stesso centro di massa. Analogamente le (12') esprimono che

b) $i$ due corpi hanno gli stessi assi principali d'inerzia. Assunti questi assi principali come assi coordinati, e chiamando $P, Q, R$ gli ordinarii momenti principali d'inerzia del corpo $C$ e $P^{\prime}, Q^{\prime}, R^{\prime}$ quelli del corpo $C^{\prime}$ le (13') dànno ovviamente

$$
Q-P=Q^{\prime}-P^{\prime}, \quad R-Q=R^{\prime}-Q^{\prime}
$$

ossia

c) $i$ tre momenti principali d'inerzia del corpo $C^{\prime}$ sono equidifferenti dai corrispondenti momenti principali del corpo C. Od anche: quando sia nota la funzione potenziale esterna di un corpo, restano per questo, determinate le differenze fra i momenti principali d'inerzia del corpo stesso.

Questi teoremi hanno interesse in quanto stabiliscono esattamente alcune proprietà che, nella teoria fisico-matematica della figura della Terra, vengono dimostrate in via approssimatc basandosi sopra espressioni approssimate della f: potenziale terrestre.

Poniamo che una superficie di livello esteriore, per un pianeta rotante uniformemente, sia quella di un ellissoide di rotazione del quale l'asse geometrico coincida con quello della rotazione. $\mathrm{E}$ intuitivo, e lo dimostreremo del resto nella $2 .^{a}$ parte di questo lavoro, che si può sempre immaginare una distribuzione di massa simmetrica sia rispetto all'asse di rotazione sia rispetto al piano dell'equatore, la quale sia compatibile colla supposta forma della superficie di livello. Per questa particolare distribuzione, il centro di massa coincide col centro dell'ellissoide, un asse principale d'inerzia coll'asse di rotazione, e l'ellissoide principale d'inerzia è, alla sua volta, di rotazione. I teoremi $a$ b) c) provano che la effettiva distribuzione della massa nell'interno del pianeta dorrà essere tale che il centro di massa coincida col centro dell'ellissoide, l'asse di rotazione sia asse principale d'inerzia, e $i$ momenti d'inerzia rispetlo a diametri nel piano equatoriale siano fra loro eguali.

In questa ipotesi che una superficie di livello esteriore terrestre sia ellissoide di rotazione, e chiamando $P$ il momento d'inerzia della massa rispetto all'asse polare, $Q$ quella rispetto ad un diametro equatoriale qualunque, ricordiamo come talune delle perturbazioni cui va soggetto il movimento della luna conducono ad una valutazione approssimata della differenza $P-Q$. E poichè è nota una relazione approssimata fra questa differenza e lo schiacciamento dell'ellissoide terrestre, il menzionato fenomeno porge un contributo alla co- 
noscenza dello schiacciamento medesimo. Che un tal contributo non involge alcuna particolare ipotesi sul modo di distribuzione della massa nell'interno della Terra, è chiara conseguenza del teorema $c$ ).

\$. Espressione della differenza fra $i$ momenti principali d'inerzia della massa terrestre. Le differenze fra i momenti principali d'inerzia sono, per quanto abbiamo dimostrato, assegnate, quando è data la f.e potenziale esterna di un corpo. Cerchiamo le espressioni generali di tali differenze e applichiamo poi il risultato al caso della Terra.

La espressione della f. potenziale di un corpo sopra un punto dell'asse $z$, a distanza $z_{1}$ dall'origine si ottiene dalla espressione generale

$$
V=\sum_{0}^{\infty} \frac{1}{p_{1}^{n+1}} \int k \rho^{n} P_{n} \cdot d \tau \quad\left(\rho_{1}>\rho\right)
$$

col porvi $P_{n}(\cos \theta)$ in luogo di $P_{n}$ e $z_{1}$ al posto di $\rho_{1}$.

Sarà

$$
V_{z}=\sum_{0}^{\infty} \frac{1}{z_{1}^{n+1}} \int k \rho^{n} P_{n}(\cos \theta) \cdot d \tau \quad\left(z_{1}>\rho\right)
$$

donde

$$
\left.\begin{array}{c}
\int k \rho^{2} P_{2}(\cos \theta) d \tau= \\
=\lim _{z_{1}=\infty} \cdot z_{1}^{2}\left(z_{1} V_{z}-\int k d \tau-\frac{1}{z_{1}} \int k \rho \cdot \cos \theta \cdot d \tau\right) .
\end{array}\right\}
$$

Chiamando $M$ la massa totale, supponendo l'origine delle coordinate nel centro di massa e gli assi coordinati coincidenti cogli assi principali d'inerzia ed osservando che

$$
\begin{aligned}
& \int k p^{2} \cdot P_{2}(\cos \theta) \cdot d \tau=\frac{1}{2} \int k p^{2}\left(3 \cos ^{2} \theta-1\right) d \tau= \\
& =\frac{1}{2} \int k\left(2 z^{2}-x^{2}-y^{2}\right) d \tau=\frac{1}{2}(P+Q-2 R),
\end{aligned}
$$

avremo dalla (14)

$$
\frac{1}{2}(P+Q-2 R)=\lim _{z_{1}=\infty} . z_{1}^{2}\left(z_{1} V_{z}-M\right) .
$$


Similmente

$$
\begin{aligned}
& \frac{1}{2}(Q+R-2 P)=\lim _{x_{1}=\infty} \cdot x_{1}^{2}\left(x_{1} V_{x}-M\right) \\
& \frac{1}{2}(R+P-2 Q)=\lim _{y_{1}=\infty} \cdot y_{1}^{3}\left(y_{1} V_{y}-M\right)
\end{aligned}
$$

donde sottraendo si hanno le cercate differenze $P-Q$, ecc.

Supponiamo $Q=R$. La penultima formola dà allora

$$
Q-P=\lim _{x_{1}=\infty} \cdot x_{1}^{2}\left(x_{1} V_{x}-M\right)
$$

Applichiamo questa formola al caso della Terra, supponendo che una superficie di livello esteriore coincida con un ellissoide di rotazione schiacciato $(a, b, b ; a<b)$, l'asse di rotazione essendo assunto come asse $x$. L'espressione della $V$ pel punto esterno $\left(x_{1} y_{1} z_{1}\right)$ è allora, come ho dimostrato in una Nota pubblicata nel $1894\left(^{*}\right)$ :

$$
\begin{gathered}
V=\left(M+\frac{2}{3} \pi \dot{k}_{0} a b^{2}\right) \frac{\operatorname{arclang} E}{\sqrt{b^{2}-a^{2}}}-\frac{2 \pi k_{0} a b^{2}}{\left(b^{2}-a^{2}\right)^{3 / 2}}(E-\operatorname{arctg} E) x_{1}^{2}- \\
-\frac{\pi k_{0} a b^{2}}{\left(b^{2}-a^{2}\right)^{3 / 2}}\left(\operatorname{arctg} E-\frac{E}{1+E^{2}}\right)\left(y_{1}^{2}+z_{1}^{2}\right)
\end{gathered}
$$

dove sono adottate le seguenti notazioni. Indicando con $\lambda$ la maggior radice della equarione

$$
\frac{x_{1}^{2}}{a^{2}+\lambda}+\frac{y_{1}^{2}+z_{1}^{2}}{b^{2}+\lambda}=1
$$

si è posto $E=\sqrt{\frac{b^{2}-a^{2}}{a^{2}+\lambda}}$. Denotando poi con $\omega$ la relocità angolare di rotazione e con $f$ la costante di attrazione, la costante $k_{0}$ che figura nella (15) è data dalla formola

$$
\frac{\omega^{2}}{2 \pi f k_{0}}=\frac{\left(3+i^{2}\right) \operatorname{arctg} i-3 i}{i^{3}}
$$

essendo $i=\frac{\sqrt{b^{2}-a^{2}}}{a}$.

(*) Sulla espressione della gravitì alla superficie del geoille supposto ellissoidlico. Rendiconti della R. Accademia dei Lincei, III, 1. ${ }^{\circ}$ sem., 1895, pag. 166 e segg.

Annali di Matenatica, Serie III, Tomo XVII. 
Per un punto dell'asse $x$ si ha

$$
\begin{gathered}
x_{1}^{2}=a^{2}+\lambda \quad E x_{1}=\sqrt{b^{2}-a^{2}} \\
x_{1} \cdot V_{x}=\left(M+\frac{2}{3} \pi k_{0} a b^{2}\right) \frac{\operatorname{arctg} E}{E}-2 \pi k_{0} a b^{2} \frac{E-\operatorname{arctg} E}{E^{3}} .
\end{gathered}
$$

Osservando che $E$ tende a zero col crescere infinito di $x_{1}$, e sviluppando in serie $\operatorname{arctg} E$ si ha

$$
x_{1} V_{x}-M=-\frac{M E^{2}}{3}+\frac{8}{45} \pi k_{0} a b^{2} \cdot E^{2}+H \cdot E^{4},
$$

dove $H$ rimane finita quando $E$ tende a zero. Quindi tenuto conto della (17):

$$
\begin{gathered}
\lim _{x_{1}=\infty}\left[x_{1}^{2}\left(x_{1} V_{: r}-M\right)\right]=-\frac{M}{3}\left(b^{2}-a^{2}\right)+\frac{8}{45}\left(b^{2}-a^{2}\right) \pi k_{0} a b^{2}= \\
=-\frac{M a^{2} i^{2}}{3}\left(1-\frac{8}{15} \frac{\pi k_{0} a}{M}\right) .
\end{gathered}
$$

Introducendo per $k_{0}$ l'espressione data dalla (16) e ricordando le (14') avremo dunque

$$
Q-P=\frac{M \alpha^{2} i^{2}}{3}\left[1-\frac{4 \omega^{2} i^{3} a b^{2}}{15 \cdot f M\left\{\left(3+i^{2}\right) \operatorname{arctg} i-3 i\right\}}\right]
$$

È questa la espressione cercata.

(Per piccoli valori di $i$, collo sviluppo in serie si ottiene, a meno di termini in $i^{4}, i^{2} \omega^{2}$ :

$$
Q-P=\frac{M a^{2}}{3}\left(i^{2}-\frac{\omega^{2} b^{2} a}{f M}\right)
$$

Il $2 .^{\circ}$ termine entro parentesi esprime prossimamente il rapporto fra la f. $^{a}$ centrifuga all'equatore e la gravità media. La relazione approssimata $\left(18^{\prime}\right)$ è ben conosciuta).

§ 7. Una estesa classe di corpi di attrazione nulla. La superficie $S$ sia una sfera di raggio $R$, il che non esige propriamente, che il corpo sia limitato da un contorno sferico; giacchè la costruzione che segue, vale anche, dal punto di vista teorico, nel caso in cui una parte dello spazio $\tau$ sia a densità nulla. 
Indichiamo ancora con $\rho_{1}, \theta_{1}, v_{1}$ le coordinate polari del punto generico dello spazio $\tau$, essendo il polo delle coordinate nel centro della sfera. Sulla sfera concentrica alla $S$ e di raggio $p_{1}(<R)$ la densità $h$ potrà essere considerata come funzione di $\theta_{1}, v_{1}$ e, salve le note restrizioni, sviluppata in serie di funzioni sferiche $Y_{n}\left(\theta_{1} v_{1}\right)$. I coefficienti di questi sviluppi varieranno con $p_{1}$; or bene noi supporremo che ciascun d'essi sia integrabile rispetto a $\dot{p}_{1}$ nell'intervallo $(0, R)$.

Porremo pertanto

$$
h=\sum_{s=0}^{\infty} Y_{s}\left(\theta_{1} v_{1}\right)
$$

La $Y_{*}$ può alla sua volta esprimersi come somma delle $2 s+1$ funzioni sferiche fondamentali $Y_{s i}$ di ordine $s$ moltiplicate per dei coefficienti indipendenti da $\theta_{1} v_{1}$ in guisa che

$$
Y_{s}=\sum_{i=1}^{2 s+1} Y_{s i}\left(\theta_{1} v_{1}\right) A_{s i}
$$

Ammetteremo dunque che i coefficienti $A_{s i}$ siano integrabili rispetto a $\rho_{1}$ nell'intervallo $(0 R)$. Avremo così

$$
h=\sum_{i=0}^{\infty} \sum_{i=1}^{2 s+1} A_{s i} Y_{s i}\left(\theta_{1} v_{1}\right)
$$

$\mathrm{E}$ per le note proprietì delle f. sferiche, indicando con $d \Omega$ l'elemento angolare di spazio e integrando su tutta la sfera di raggio 1 :

$$
\int_{4 \pi} h P_{n} . d \Omega=\frac{4 \pi}{2 n+1} \sum_{i=1}^{2 n+1} A_{n i} Y_{n i}(\theta v) \text {. }
$$

Sostituendo nella (2) ove poniamo $d \tau=\rho^{2} . d \rho \cdot d \Omega$

$$
\int_{0}^{R} \int_{n \pi}^{n} h \rho^{n+2} P_{n} \cdot d \Omega \cdot d \rho=\frac{4 \pi}{2 n+1} \sum_{i=1}^{2 n+1} Y_{n i}(\theta v) \int_{0}^{R} A_{n i} \rho^{n+2} \cdot d \rho=0 .
$$

E poichè per l'annullarsi di una funzione sferjca di ordine $n$ è necessario che si annullino i coefficienti di ciascuna delle f. ${ }^{1}$ sferiche fondamentali che la costituiscono, avremo come condizione necessaria e sufficiente pel verificarsi della (2)

$$
\int_{i}^{R} \rho^{n+2} A_{m i} \cdot d \rho=0
$$


Giascuno dei coefficienti dello sviluppo di $h$ è pertanto legato alla sola condizione (20). Uno dei più semplici modi particolari di assegnare questi coefficienti sarà, p. es., porre:

$$
A_{n i}=\alpha+\beta \frac{\rho}{R}+\gamma \frac{\rho^{2}}{R^{2}}+\cdots
$$

dove le costanti $\alpha \beta \gamma \ldots$ sono legate alla sola condizione

$$
\frac{\alpha}{n+3}+\frac{\beta}{n+4}+\frac{\gamma}{n+5}+\cdots=0
$$

che, in questo caso, equivale alla (20) (*).

Se dunque in una porzione sferica $S$ dello spazio occupato da un corpo $C$, si muta la distribuzione della massa, col dare alla densità un incremento espresso dalla (19), ove i coefficienti $A_{n i}$ soddisfacciano alla (20), l'attrazione del corpo $C$ all'esterno non sarà alterata.

§ 8. Si può ancora porre la condizione che lo spostamento di mosso avvenga secondo il raggio vettore, in guisa che non si alteri la quanlità totale di materia contenula in ogni cono avente il vertice nel centro della sfera. facile vedere che, in questo caso, i coefficienti $A$, oltrechè alla (20) debbono anche soddisfare alla

$$
\int_{i}^{R} A_{n i} \rho^{2} \cdot d \rho=0 \text {. }
$$

Ovvero si può chiedere che $i$ mutamenti di distribuzione avvengano nelle immediate vicinanze della superficie $S$.

Basterà allora, indicando con $R_{1}$ un valore compreso fra 0 ed $R$ e abbastanza prossimo ad $R$, dare agli $A_{2 i}$ valore nullo per $p$ compreso fra 0 ed $R_{1}$, e, nell'intervallo $\left(R_{1}, R\right)$ esprimere gli stessi $A_{n i}$ mediante funzioni integrabili di $\rho$ soggette alla sola condizione

$$
\int_{\dot{n}_{1}}^{R} A_{n i} \rho^{n+2} \cdot d \rho=0 .
$$

$\left(^{*}\right)$ E facile vedere che occorre che la $a$ sia nulla per tutti i valori di $n$ salvo per $n=0$, affinchè la densità $h$ abbia un valore unico e determinato nel centro della sfera. 
§9. Le deduzioni dei due precedenti paragrafi non esigono, come già si è osservato che il corpo $\tau$ riempia tutto lo spazio limitato dalla sfera $S$, sicchè la superficie limitante il corpo può avere una forma molto generale diversa dalla sferica, senza che le formole trovate perdano, teoricamente, il loro valore. Ma è da osservare che, in questo caso, per ogni valore di p compreso in un tratto finito fra zero ed $R$, i valori della densità $h$ sulla sfera di raggio $\rho$ presentano necessariaménte delle discontinuità. 'La rappresentazione della $h$ mediante lo sviluppo (19) può ancora ottenersi; ma gli sviluppi di funzioni discontinue in serie di funzioni sferiche, se costituiscono un bel campo di indagine per l'analisi pura, non hanno alcuna pratica utilità nello studio numerico dei fatti naturali.

$\S 10$. Altro metodo per rappresentare corpi di attrazione nulla. Consideriamo una funzione qualsiasi $f(x, y, z)$ dei punti dello spario $\tau$, limitato dalla superficie $S$, la quale sia finita e continua insieme colle sue derivate $1 .^{\circ}$, ed abbia le derivate $2 .^{c}$ finite ed integrabili in ogni punto di $\tau$ e noniamo che nei punti della superficie $S$ si abbia

$$
f=0, \quad \frac{\partial f}{\partial n}=0 .
$$

Posto

$$
h=\Delta_{2} f
$$

un corpo situato entro lo spazio t e la cui densità nel punto generico sia $\Delta_{2} f$, sarà di attrazione esterna nulla. Infatti per la nota formola di Gresen si ha, per un punto qualumque esterno alla superficie $S$

$$
0=\int_{\tau} \Delta_{2} f \frac{d \tau}{r}+\int_{S}\left(f \frac{\partial \frac{1}{r}}{\partial n}-\frac{1}{r} \frac{\partial f}{\partial n}\right) d S .
$$

E poicliè, per le ipotesi fatte, il $2 .^{\circ}$ integrale è nullo, risulta pure nulla la f. ${ }^{\text {e }}$ potenziale del corpo sopra ogni punto esterno. Reciprocamente, se con $f$ si indica la funzione potenziale, in un punto interno, di un corpo che non esercita alcuna attrazione all'esterno, dovrà, nei punti della superficie, la $f$ soddisfare alle condizioni (22), e la densità in un punto generico sarà, a meno del divisore costante $-4 \pi$, espressa dalla (22).

La ricerca dei corpi di attrazione nulla, relativi allo spazio $\tau$, può dunque 
ridursi alla ricerca di tutte quelle funzioni $f$ che entro $\tau$ godono delle menzionate condizioni di regolarità, e sulla $S$ godono delle proprietà (29).

Poniamo in particolare che la $S$ sia un ellissoide $(a, b, c)$. Posto

$$
1-\frac{x^{2}}{a^{2}}-\frac{y^{2}}{b^{2}}-\frac{z^{2}}{c^{2}}=2 k
$$

$\mathrm{e}$ indicando con $\varphi(x, y, z)$ una funzione che, sulla superficie e nell'interno dell'ellissoide, goda delle dette condizioni di regolarità, potremo porre

$$
f(x, y, z)=k^{2} \cdot \varphi(x, y, z)
$$

con che le condizioni (22) saranno soddisfatte. La densità del corpo nel punto $(x, y, z)$ sarà allora

$$
h=\Delta_{2} f=\left(D^{2}-k A\right) 2 \varphi-4 k \mathbf{\Sigma} \frac{x}{a^{2}} \frac{\partial \varphi}{\partial x}+k^{2} \Delta_{2} \varphi,
$$

dove

$$
D^{2}=\frac{x^{2}}{a^{4}}+\frac{y^{2}}{b^{4}}+\frac{z^{2}}{c^{4}}, \quad A=\frac{1}{a^{2}}+\frac{1}{b^{2}}+\frac{1}{c^{2}},
$$

e nella ipotesi più semplice, $\varphi=$ cost.

$$
h=\alpha\left(\frac{x^{2}}{a^{4}}+\frac{y^{2}}{b^{4}}+\frac{z^{2}}{c^{4}}\right)-\frac{\alpha}{2}\left(1-\frac{x^{2}}{a^{2}}-\frac{y^{2}}{b^{4}}-\frac{z^{2}}{c^{4}}\right)\left(\frac{1}{a^{2}}+\frac{1}{b^{2}}+\frac{1}{c^{2}}\right)
$$

dove $\propto$ è una costante.

Ritornando al caso in cui la $S$ sia una sfera di raggio $R$, possiamo ritrovare nuovamente il risultato del $\$ 8$. Poniamo

$$
f=\sum_{\varepsilon=0}^{\infty} B_{s} Y_{s}
$$

dove $Y_{*}$ è funzione sferica dell'ordine $s, e B_{x}$ è funzione del raggio vettore $p$ che ammette derivate $1 . .^{\circ}$ finite e continue e derirate $2 .^{\circ}$ finite ed integrahili, per $p$ compreso fra 0 ed $R$, e si suppone che, per $\hat{p}=R$, si abbia:

$$
B_{s}=0, \quad \frac{d B_{s}}{d \rho}=0
$$

Avremo, ricordando la espressione del $\Delta_{2}$ in coordinate polari:

$$
\Delta_{s}\left(B, Y_{s}\right)=\frac{Y_{s}}{\rho^{2}} \frac{d}{d \rho}\left(\rho^{2} \frac{d B_{s}}{d \rho}\right)+\beta_{\rho^{2}} \frac{B_{s}}{\operatorname{sen} \theta} \frac{\partial}{\partial \theta}\left(\operatorname{sen} \theta \frac{\partial Y_{s}}{\partial \theta}\right)+\frac{B_{s}}{\rho^{2} \operatorname{sen}^{2} \theta} \frac{\partial^{2} Y_{s}}{\partial v^{2}} .
$$


Ovvero, tenuto conto della equazione differenziale cui la $Y_{s}$ soddisfa

Poniamo

$$
\Delta_{2}\left(B_{s} Y_{s}\right)=\frac{Y_{s}}{\rho^{2}}\left\{\frac{d}{d \rho}\left(\rho^{2} \frac{d B_{s}}{d \rho}\right)-s(s+1) B_{s}\right\} .
$$

sarà

$$
\frac{1}{\rho^{2}} \frac{d}{d \rho}\left(\rho^{2} \frac{d B_{s}}{d \rho}\right)-\frac{s(s+1)}{\rho^{2}} B_{s}=A_{s}
$$

$$
h=\sum_{0}^{\infty} A_{s} Y_{s}
$$

la densità, nel punto generico, di un corpo d'attrazione esterna nulla contenuto entro la sfera di raggio $R$.

Con due successive integrazioni per parti si ha, tenuto conto delle (22):

$$
\int_{0}^{n} \rho^{s} \frac{d}{d \rho}\left(\rho^{2} \frac{d B_{s}}{d \rho}\right) d \rho=-s \int_{i}^{R} \rho^{n+1} \frac{d B_{s}}{d \rho} d \rho=s(s+1) \int_{j}^{k} \rho^{s} . B_{s} . d \rho .
$$

Sicchè la (26) moltiplicata per $p^{x+t^{2}}$ è integrata rispetto a $p$ fra zero ed $R$ dà :

$$
\int_{0}^{R} \rho^{s+2} A_{s} \cdot d \rho=0
$$

ossia, di nuovo, la condizione già trovata nel $\$ 7$.

$\S 11$. Altri corpi ellissoidici di attrazione nulla. Una classe molto generale di corpi di attrazione nulla a contorno ellissoidico si è ottenuta colla formola (24) del precedente paragrafo. Possiamo ottenerne altre valendoci del teorema di LAPLACE relativo alla attrazione di due ellissoidi omofocali, dal quale si deduce che una distribuzione per strati ellissoidici omogenei omofocali (di spessore finito o infinitesimo) esercita attrazione nulla all'esterno, semprechè la massa totale sia nulla.

Si considerino infatti tre ellissoidi omofocali $E, E_{1}, E_{2}$ dei quali il primo esteriore, il terzo interno agli altri due. Supponendoli successivamente riempiuti di materia omogenea e dette $M, M_{1}, M_{2}$ le masse, $V, V_{1}, V_{2}$ le f. ${ }^{i}$ potenziali rispettive sopra uno stesso punto esterno $P$, si deduce dal teorema di Laplace

$$
\frac{V_{1}}{V}=\frac{M_{1}}{M}, \quad \frac{V_{2}}{V}=\frac{M_{z}}{M}
$$


e quindi, posto:

risulta

$$
V_{2}-V_{1}=\Delta V, \quad M_{2}-M_{1}=\Delta M
$$

$$
\Delta V=\frac{V}{M} \cdot \Delta M
$$

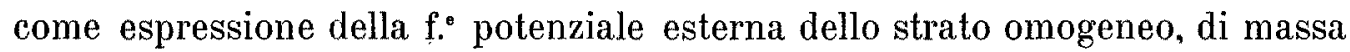
$\Delta M$ compreso fra gli ellissoidi $E_{1}, E_{2}$. Il rapporto $V: M$ dipende unicamente dai semiassi dell'ellissoide esterno $E$, oltrechè, s'intende, dalle coordinate del punto potenziato $P$. Se, pertanto, supponiamo lo spazio interno all'ellissoide $E$ occupato da una massa costituita da strati omogenei limitata da ellissoidi omofocali, la funzione potenziale di questa massa sopra un punto esterno sarà

$$
v=m \frac{V}{M}
$$

dove $m$ è la grandezza della massa totale. Sarà necessario e sufficiente che questa sia nulla perchè sia pur nulla $v$. Indichiamo con $\lambda$ la maggior radice dell'equazione

$$
\frac{x^{2}}{a^{2}+\lambda}+\frac{y^{2}}{b^{2}+\lambda}+\frac{z^{2}}{c^{2}+\lambda}=1
$$

$\left(a<b<c ; \lambda>-a^{2}\right)$ dovrà la densità $h$ variare solo con $\lambda$.

Posto $h=\varphi(\lambda)$ (dove $\varphi$ potrà anche presentare discontinuità ordinarie, e mantenersi costante per tratti finiti dell'intervallo $\left(0,-a^{2}\right)$ ), la condizione: $m=0$ si esprime, com'è facile verificare, con

$$
\int_{-a^{2}}^{0} \varphi(\lambda) \cdot d \sqrt{\left(a^{2}+\lambda\right)\left(b^{2}+\lambda\right)\left(c^{2}+\lambda\right)}=0
$$

ovvero con

$$
\int_{-a^{2}}^{0} \sqrt{\left(a^{2}+\lambda\right)\left(b^{2}+\lambda\right)\left(c^{2}+\lambda\right)}\left|\frac{1}{a^{2}+\lambda}+\frac{1}{b^{2}+\lambda}+\frac{1}{c^{2}+\lambda}\right| \varphi(\lambda) \cdot d \lambda=0 .
$$

\$12. Un terzo modo di costruzione di corpi di attrazione nulla. Si consideri un corpo sferico di raggio $c$, composto di croste sferiche omogenee; sia $\varphi(r)$ la densilà a distanza $r$ dal centro. Se è soddisfatta la condizione

$$
\int_{0}^{c} r^{2} \varphi(r) \cdot d r=0
$$


sarà nulla la massa totale d'un tal corpo sferico, e quindi nulla la funzione potenziale di esso sopra ogni punto esterno. Chiameremo sferula un corpo fittizio di tale costituzione. Distribuendo quante si vogliano sferule entro uno spazio finito e considerando un corpo la cui densità in un punto sia la somma algebrica delle densità delle sferule cui quel punto appartiene, la funzione potenziale di questo corpo sarà nulla per ogni punto esterno ad esso.

Dato ora lo spazio finito $\tau$, limitato dalla superficie $S$, consideriamo entro questo spazio distribuite infinite sferule in guisa che nessun punto di alcuma di esse sia esterno ad $S$.

Pèr una qualunque di tali sferule, la funzione o $(r)$ si porrù uguale a

$$
b \cdot f(r, c) \cdot d \tau
$$

ove $b$ e $c$ siano funzioni delle coordinate del centro della sferula e si suppone, ben inteso, verificata la condizione

$$
\int_{0}^{c} r^{2} f(r, c) \cdot d r=0
$$

Si potranno supporre i valori del raggio $c$ abbastanza piccoli perchè esista una porzione finita $\tau_{2}$ (limitata nella ligura dalla superficie $S_{2}$ ) dello spazio $\tau$, i punti della quale non appartengano ad alcuna delle sferule, che toccano la superficie esterna $S(*)$.

Supponiamo $c=$ costante. Allora per un punto qualunque $P_{2}$ dello spazio $\tau_{2}$, considerato il solido sferico $T$ di raggio $c$ e centro

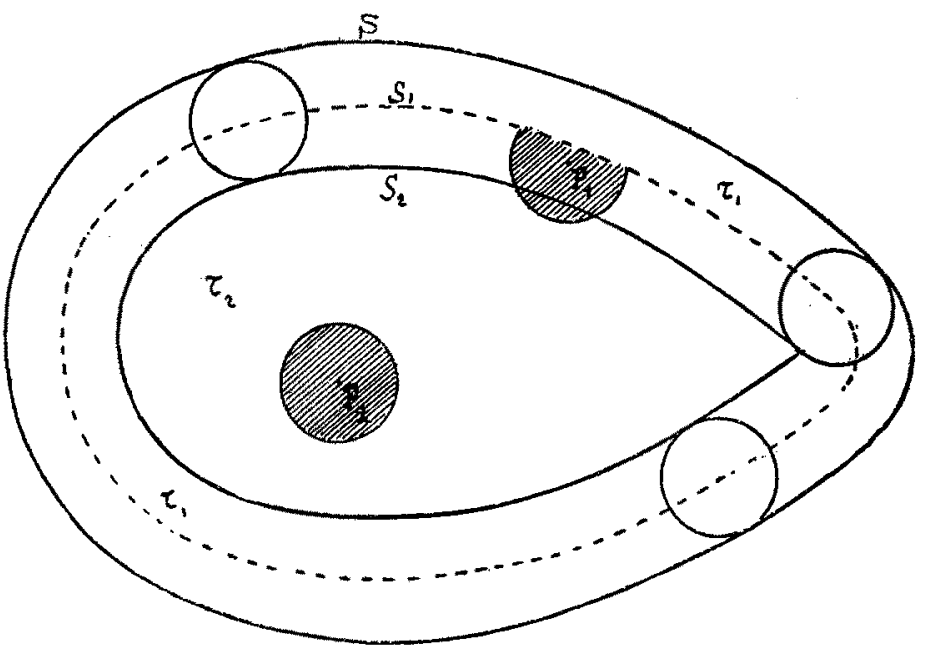
$P_{2}$, in ogni punto di questo solido vi sarà il centro di una sferula che darà

(*) Aćcadrà, ove le curvature delle sezioni normali della $S$ siano superiori a quelle delle sferule che la toccano, che restino porzioni dello spazio $\tau$, $i$ punti delle quali non appartengano ad alcuna sferula. Ma ciò potrà evitarsi assumendo abbastanza piccoli i raggi $c$, se le dette curvature non superano un limite finito.

Annali di Matematica, Serie III, Tomo XVII. 
un contributo alla densità del punto $P_{2}$. La densità nel punto $P_{2}$ sarà quindi

$$
h=\int_{T} b \cdot f(r, c) \cdot d \tau
$$

ove $r$ è la distanza dell'elemento $d \tau$ dal punto $P_{2}$.

Invece per un punto $P_{1}$ del rimanente spazio $\tau_{1}$ (compreso fra le superficie $S_{2}$ ed $S$, consideriamo la superficie $S_{1}$ luogo dei centri delle sferule che toccano la $S$; alla (29) andrà sostituita la

$$
h=\int_{T^{\prime}} b f(r, c) \cdot d \tau
$$

dove con $T^{\prime}$ si intende lo spazio compreso fra la superficie $S_{1}$ e quella porzione (tratteggiata in figura) di sfera di centro $P_{1}$ e raggio $c$ che è interna ad $S_{1}$.

Poniamo nella (29) $d \tau=r^{2} . d r . d \Omega$ essendo $r$ la distanza del punto generico dal punto $P_{2}$ e $d \Omega$ l'elemento angolare di spazio intorno a $P_{2}$. Avremo

$$
h=\int_{0}^{c} r^{2} \cdot d r \cdot f(r, c) \int_{4 \pi} b \cdot d \Omega=4 \pi \int_{0}^{c} r^{2} \cdot M \cdot f(r, c) \cdot d r
$$

ove con $M$ indichiamo il valore medio fra quelli che la funzione $b$ assume nei punti di una sfera di centro $P_{2}$ e raggio $r$. Supponiamo che la funzione $b$ sia regolare e tale che il valore del suo parametro differenziale d'ordine $2 n+2$ sia o nullo o trascurabile. Allora, come ho dimostrato in altro mio lavoro $\left(^{*}\right)$, si arrà

$$
M=b_{0}+\sum_{i=1}^{n} \frac{r^{2 i}}{(2 i+1) !}\left(\Delta_{2 i} b\right)_{0}
$$

dove $b_{0}$ è il valore di $b$ nel punto $P_{2}$ e $\left(\Delta_{2 i} b\right)_{0}$ è il valore del parametro differenziale d'ordine $2 i$ nel punto stesso. Sostituendo nella (30) e tenuto conto della (28) avremo:

$$
h=4 \pi \sum_{i=1}^{n} \frac{K_{i}}{(2 i+1) !}
$$

dove le $K_{i}$ sono costanti date da

$$
K_{i}=\int_{0}^{e} r^{2 i+2} f(r, c) \cdot d r
$$

${ }^{*}{ }^{*}$ Rendiconti della $R$. Accademia dei Lincei, XVIII, $1 .^{\circ}$ sem., serie $5 .^{\mathrm{a}}$, pag. 182 (febbrajo 1909). 
$\S 13$. In particolare se la $b$ è funzione armonica in $\tau$, risulta $h=0$. Se, più generalmente, $\Delta_{2} b$ è costante $(=a)$, e quindi son nulli i parametri differenziali d'ordine superiore, risulterà pure $h=$ costante, ossia

$$
h=\frac{2 \pi a}{3} \int_{0}^{c} r^{4} f(r, c) d r .
$$

(Una delle più semplici forme che può assumere la $f(r, c)$ compatibilmente colla condizione $(28)$, è

$$
f(r, c)=c-\frac{4}{3} r
$$

La $\left(30^{\prime}\right)$ dà allora $\left.h=-\frac{2 \pi a c^{6}}{135}\right)$.

Segue da ciò che si può sempre in un corpo d'attrazione nulla supporre costante la densità per una porzione finila dello spazio occupato dal corpo.

$\mathrm{E}$ di qui si deduce che, dato un corpo $C$ omogeneo, di densità $k_{0}$, si può generalmente (salve le restrizioni che stabiliremo riguardo alla superficie esterna) considerare uno strato materiale esterno di spessore finito costante, aderente al corpo, e di densità variabile $k^{\prime}$ la cui funzione potenziale esterna sia eguale a quella del corpo $C$ medesimo. Per dimostrarlo indichiamo con $\tau_{2}$ lo spazio occupato da $C$, con $S_{2}$ la superficie che lo limita, e consideriamo lo' spazio $\tau_{1}$ i cui punti appartengono alle infinite sfere di raggio costante $c$ che tocano esternamente la $S_{2}$ senza penetrare nello spazio $\tau_{2}$. Se la $S_{2}$ è convessa, o se, presentando delle regioni di concavità, la curvatura delle sezioni normali che volgono la concavità all'esterno non eccede, in valore assoluto un certo limite finito, si potrà sempre assumere il raggio $e$ abbastanza piccolo perchè ogni punto della $S_{2}$ sia punto di contatto di una sfera di raggio $c$ tutta esterna al corpo $\tau_{2}$. In tal caso non vi sarà alcuna soluzione di continuità fra- lo spazio $\tau_{1}$ e il $\tau_{2}$ e potremo dire che il $1 .^{\circ}$ aderisce al $2 .^{\circ}$; di più la superficie $S$ limitante esternamente $\tau_{1}$ risulterà parallela alla $S_{2}$ e a distanza costante $2 c$ da questa.

Entro lo spazio $\tau_{1}+\tau_{2}$ distribuiamo, come nel paragrafo precedente, un sistema continuo di sferule di raggio $c$, assumendo $b$ uguale ad una funzione di $2{ }^{\circ}$ grado delle coordinate, p. es.

$$
b=\frac{a}{6}\left(x^{2}+y^{2}+z^{2}\right) \quad(a=\text { costante }) .
$$


Sarà $\Delta_{a} b=a$. Nell'interno di $\tau_{z}$ risulterà la densità $h$ data dalla formola $\left(30^{\prime}\right)$ e quindi costante. Assumeremo la costante $\alpha$ in guisa che risulti $h=k_{0}$. Nello spazio $\tau_{1}$ la densità $h^{\prime}$ risulterà espressa dalla $\left(29^{\prime}\right)$ e sarà variabile da punto a punto. Posto $k^{\prime}=-h^{\prime}$ ̀̀ chiaro che una massa distribuita nello spazio $\tau_{1}$ con densità generica $k^{\prime}$, eserciterà all'esterno una attrazione úguale a quella esercitata dalla massa omogenea $k_{0}$ contenuta nello spazio $\tau_{2}$.

Ricerca di alcuni modi dI Distribuzione della massa terhestre, CHE SONO COMPATIBILI COLLA SUPERFICIE DI LIVELLO ELLISSOIDIGA.

$\$ 14$. Funzione potenziale esterna della attrazione terrestre nel caso in cui una superficie di livello esteriore sia ellissoide di rotazione schiacciato: $E(a, b, b ; a<b)$. Già abbiamo ricordato al $\$ 6$ quale sia l'espressione, in termini finiti, di questa funzione potenziale $V$. Qui giova piuttosto valersi di un'altra più succinta espressione. Nella citata Nota del 1894 ho dimostrato come quella funzione possa ottenersi come somma della f. ${ }^{\circ}$ potenziale $V_{1}$ di uno strato ellissoidico omotetico e di quella $V_{2}$ di un ellissoide omogeneo di densità $k_{0}$ opportunamente fissata; in guisa che

$$
V=\frac{u}{2} \int_{i}^{\infty} \frac{d s}{\sqrt{\bar{R}}}+\pi k_{0} a b^{2} \int_{i}^{\infty} \frac{h}{\sqrt{R}} d s=V_{1}+V_{2}
$$

dove

$$
h=1-\frac{x^{2}}{a^{2}+s}-\frac{y^{2}+z^{2}}{b^{2}+s}, \quad R=\left(a^{2}+s\right)\left(b^{2}+s\right)^{2}
$$

(l'asse $x$ è assunto come asse geometrico di rotazione dell'ellissoide, e insieme come asse di rotazione della terra).

La 'usiliaria $k_{0}$ è data dalla relazione già ricordata a $\$ 6$

$$
\frac{\omega^{2}}{2 \pi f k_{0}}=\frac{\left(3+i^{2}\right) \operatorname{arctg} i-3 i}{i^{3}} ; \quad\left(i=\frac{\sqrt{b^{2}}-a^{2}}{a}\right)
$$

$\lambda$ è la maggior iadice dell'equazione

$$
\frac{x^{2}}{a^{2}+\lambda}+\frac{y^{2}+z^{2}}{b^{2}+\lambda}=1
$$


Quanto alla $\mu$, essa è determinata dalla condizione che sia

$$
\lim _{\lambda=\infty} \sqrt{\lambda} V=M I
$$

ove $M$ è la massa totale della Terra. E poichè

$$
\lim _{\lambda=\infty} \sqrt{\lambda} \int_{\lambda}^{\infty} \frac{d s}{\sqrt{\bar{R}}}=2, \quad \lim _{\lambda=\infty} i \lambda \int_{\lambda}^{\infty} \frac{h}{\sqrt{\bar{R}}} d s=\frac{4}{3}
$$

risulta

$$
\mu=N-\frac{4}{3} \pi k_{0} a b^{2}
$$

Il valore numerico della $k_{0}$ può, nel caso della Terra, calcolarsi all'ingrosso osservando che se si indica con $k_{m}$ la densità media della Terra, e con $G_{i i}$ la gravita media superficiale, si la prossimamente

$$
2 \pi f=\frac{3}{2} \frac{a_{m}}{a k_{m}}, \quad \frac{\omega^{2} a}{G_{m}}=\frac{1}{288}
$$

donde $\frac{\omega^{2}}{2 \pi f}=\frac{k_{m}}{4392}$. D'altra parte, se si adotta il valore Besseliano dello schiacciamento terrestre, il secondo membro della (16) assume prossimamente il valore $1: 537$. Ne segue

$$
k_{i}=1,3 k_{m} \quad \text { circa. }
$$

La y. definita dalla (33) è dunque negativa, ed è uguale in valore assoIuto a circa tre decimi della massa totale terrestre.

\$15. Distribuzione corrispondente al termine $V_{2}$. Avendo già, nella prima parte di questo lavoro, trattato con una certa generalita il problema delle possibili alterazioni che può subire una distribuzione spaziale di massa nell'interno di una superficie chiusa $S$ senza che resti alterata la f. potenziale esterna, basterà ora che cerchiamo una, o qualcuna, delle distribuzioni compatibili colla espressione (31) della f. potenziale dell'attrazione terrestre.

Quanto al termine $V_{2}$, ad esso si può far corrispondere o una distribuzione omogenea con densità $k_{0}, 0$, più generalmente, una distribuzione per strati ellissoidici omofocali di spessore finito o infinitesimo (\$11) con den- 
sità variabile da strato a strato e soggetta alla sola condizione che la massa totale sia $\frac{4}{3} \pi k_{0} a b^{2}$.

\$16. Il termine $V_{1}$. Poichè dalle effettive distribuzioni di massa vogliamo naturalmente escludere quelle per le quali la densità spaziale è in qualche punto infinita, dobbiamo sostituire allo strato superficiale, del quale il termine $V_{1}$ esprime la funzione potenziale esterna, una massa distribuita, con densità spaziale finita, in tutto o in parte dello spazio racchiuso dall'ellissoide $E$.

Si tratta di trovare una funzione $V_{i}$ finita e continua, insieme colle sue derivate $10^{\circ}$ e colle derivate 2.' finite ed integrabili, all'interno di $E$, tale che nei punti di $E$ si abbia

$$
V_{i}=V_{1}, \quad \frac{\partial V_{i}}{\partial r}=\frac{\partial V_{1}}{\partial r}
$$

indicando con $r$ una delle coordinate.

Una classe estesa di soluzioni si ottiene ponendo

$$
V_{i}=A-\frac{u}{2 a b^{2}} \Phi(\lambda)
$$

love $A$ è una costante $=\frac{\mu}{2} \int_{0}^{\infty} \frac{d s}{\sqrt{\bar{R}}}$ e $\Phi(\lambda)$ soddisfà alle condizioni

$$
\Phi(0)=0, \quad \Phi^{\prime}(0)=1,
$$

e a condizioni di regolarità che fra poco diremo. La $1 .^{a}$ delle (34) è evidentemente verificata. Quanto alle altre, osserviamo che dalla (35) si deduce:

$$
\frac{\partial V_{i}}{\partial x}=-\frac{\mu}{2 a b^{2}} \Phi^{\prime}(\lambda) \frac{\partial \lambda}{\partial x},
$$

e dalla espressione di $V_{1}$

$$
\frac{\partial V_{1}}{\partial x}=\frac{-\mu}{2 \sqrt{\left(a^{2}+\lambda\right)\left(b^{2}+\lambda\right)^{2}}} \frac{\partial \lambda}{\partial x}
$$

Quindi nei punti di $E(\lambda=0)$

$$
\frac{\partial V_{i}}{\partial x}=\frac{\partial V_{1}}{\partial x} .
$$


La densità corrispondente all'espressione (35) della $V_{i}$ è

$$
k_{1}=-\frac{1}{4 \pi} \Delta_{2} V_{i}=\frac{\mu}{8 \pi a b^{2}} \cdot \Delta_{2} \Phi(\lambda) .
$$

Se al termine $V_{2}$ della (31) si fa corrispondere una distriluzuione omogenea con densità $k_{0}$ e si osserva che la (33) può scriversi

$$
\mu=-\frac{4}{3} \pi\left(k_{n}-k_{m}\right) a b^{2}
$$

risulterà la densità tolale, nel punto generico, data da:

$$
\left.\begin{array}{c}
k=k_{0}+k_{1}=k_{0}-\frac{k_{0}-k_{m}}{6} \Delta_{2} \Phi(\lambda)= \\
=k_{m}\left|1,3-0,05 \cdot \Delta_{2} \Phi(\lambda)\right|
\end{array}\right\}
$$

poichè, come si è visto, $k_{0}=1,3 k_{m}$.

Si ha poi

$$
\Delta_{2} \Phi(\lambda)=\Phi^{\prime}(\lambda) \cdot \Delta_{2} \lambda+\Phi^{\prime \prime}(\lambda) \cdot \Delta_{1} \lambda
$$

dove le espressioni di $\Delta_{1} \lambda, \Delta_{2} \lambda$ si deducono senza difficoltà dalla (32) considerandovi $\lambda$ come funzione di $x, y, z$. Posto

si ha

$$
\frac{x^{2}}{\left(a^{2}+\lambda\right)^{2}}+\frac{y^{2}+z^{2}}{\left(b^{2}+\lambda\right)^{2}}=P
$$

$$
\begin{gathered}
\frac{\partial \lambda}{\partial x}=\frac{2 x}{\left(a^{2}+\lambda\right) P}, \quad \Delta_{1} \lambda=\frac{4}{P} \\
\Delta_{2} \lambda=\frac{2}{P}\left(\frac{1}{a^{2}+\lambda}+\frac{1}{b^{2}+\lambda}+\frac{1}{c^{2}+\lambda}\right)=\frac{\Delta_{1} \lambda}{2}\left(\frac{1}{a^{2}+\lambda}+\frac{1}{b^{2}+\lambda}+\frac{1}{c^{2}+\lambda}\right)
\end{gathered}
$$

e sostituendo nella espressione di $\partial V_{i}: \partial x$

$$
\frac{\partial V_{i}}{\partial \cdot x}=-\frac{\mu \cdot x}{4 a b^{2}\left(a^{2}+\lambda\right)} \Phi^{\prime}(\lambda) \cdot \Delta_{1} \lambda .
$$

Affinchè la soluzione (35) sia ammissibile, occorre che le espressioni (39) (40) rimangano finite in tutto lo spazio interno ad $E$. Questa condizione limita di molto la scelta della funzione $\Phi$. Per vederlo ricordiamo come si comportino le funzioni $\Delta_{1} \lambda, \Delta_{2} \lambda$ nell'interno dell'ellissoide. 
$\$ 17$. Consideriamo più generalmente un ellissoide a tre assi $(a<b<c)$, e siostituiamo, per un momento, alla lettera $\lambda$ la $\rho_{1}$, chiamando $p_{1}, p_{2}, p_{8}$ le ordinarie coordinate ellittiche, radici della equazione

$$
\begin{gathered}
\frac{x^{2}}{a^{2}+p}+\frac{y^{2}}{b^{2}+p}+\frac{z^{2}}{c^{2}+p}=1, \\
+\infty>p_{1} \supseteq-a^{2} \supseteq p_{2} \supseteq-b^{2} \supseteq p_{3} \supseteq-c^{2} .
\end{gathered}
$$

Indicando con

$$
d s^{2}=H_{1}^{2} \cdot d \rho_{1}^{2}+H_{2}^{2} \cdot d \rho_{2}^{2}+H_{3}^{2} \cdot d \rho_{3}^{2}
$$

il quadrato dell'elemento lineare dello spazio in coordinate ellittiche, abbiamo

$$
H_{1}^{2}=\frac{1}{4} \frac{\left(p_{1}-p_{2}\right)\left(p_{1}-p_{3}\right)}{\left(a^{2}+p_{1}\right)\left(b^{2}+p_{1}\right)\left(c^{2}+p_{1}\right)}
$$

ed analoghe espressioni per $H_{2}, H_{3}$. Quindi

$$
\begin{gathered}
\Delta_{1} p_{1}=\frac{1}{H_{1}^{2}}=\frac{4\left(a^{2}+p_{1}\right)\left(b^{2}+p_{1}\right)\left(c^{2}+p_{1}\right)}{\left(\rho_{1}-p_{2}\right)\left(p_{1}-p_{3}\right)} \\
\Delta_{2} p_{1}=\frac{1}{H_{1} H_{2} H_{3}} \frac{\partial}{\partial \rho_{1}}\left(\frac{H_{2} H_{3}}{H_{1}}\right)= \\
=\frac{2}{\left(p_{1}-p_{2}\right)\left(\rho_{1}-p_{3}\right)}\left[\left(a^{2}+p_{1}\right)\left(b^{2}+\rho_{1}\right)+\left(b^{2}+p_{1}\right)\left(c^{2}+p_{1}\right)+\left(c^{2}+p_{1}\right)\left(a^{2}+p_{1}\right)\right] .
\end{gathered}
$$

Poichè $a$ si suppone differente da $b$ (mentre $b$ in particolare potrà essere uguale a $c$, in guisa che il nostro ragionamento comprende anche il caso dell'ellissoide di rotazione considerato nei precedenti paragrafi), sarà $p_{1}>p_{3}$. Si ha poi generalmente $p_{1}>p_{2}$, salvo nei punti della ellisse focale dove $p_{1}=p_{2}=-a^{2}$. Sicche la $\Delta_{2} p_{1}$ diviene infinita nei punti dell'ellisse stessa, e soltanto in quei punti.

Quanto a $\Delta_{1} \rho_{1}$, questa quantità rimane sempre finita. Nei punti esterni al piano $y z$ e nella regione al piano stesso che è esterna all'ellisse focale si ha $\rho_{1}>p_{2}, p_{1}>-a^{2}$ e quindi ivi $\Delta_{1} p_{1}$ è finita e differente da zero. Nei punti interni alla ellisse stessa $\rho_{1}=-a^{2}>\rho_{2}$ e quindi $\Delta_{1} \rho_{1}=0$. Nei punti della ellisse finalmente per essere $p_{1}=p_{2}=-a^{2}$ si ha dalla (4:3)

$$
\lrcorner_{1} p_{1}=\frac{4\left(b^{2}+p_{1}\right)\left(c^{2}+p_{1}\right)}{p_{1}-p_{3}}=\frac{4\left(b^{2}-a^{2}\right)\left(c^{2}-a^{2}\right)}{-\left(a^{2}+p_{3}\right)} .
$$


E poichè

sarà

$$
c^{2} \supseteq-p_{3} \supseteq b^{2}
$$

$$
4\left(b^{2}-a^{2}\right)<\Delta_{1} p_{1}<4\left(c^{2}-a^{2}\right) .
$$

Nel caso dell'ellissoide di rotazione $\Delta_{1} p_{1}=4\left(b^{2}-a^{2}\right)$ nei punti dell'ellisse focale.

Osserviamo poi che, chiamando $\alpha$ il $10^{\circ}$ coseno di direzione della normale all'ellissoide (32) nei punti $x, y, z$, si ha

donde

$$
\alpha=\frac{x}{\left(a^{2}+\lambda\right) \sqrt{P}}=\frac{x}{2\left(a^{2}+\lambda\right)} \sqrt{\Delta_{1} \lambda},
$$

$$
\frac{x}{\left(a^{2}+\lambda\right)} \Delta_{1} \lambda=2 \alpha \sqrt{\Delta_{1} \lambda}
$$

Ne segue, per quel che ora si è detto intorno al $\Delta_{1} p_{1}$, che, se $\Phi^{\prime}(\lambda)$ si mantiene finita per $\lambda$ compreso fra 0 e $-a^{2}$, tali si manterranno pure le derivate prime della $V_{i}$ [formola (40)].

\$ 18. Differenza fra la sfera e gli ellissoidi, riguardo alle soluzioni del. tipo (35). Nel caso della sfera il $\Delta_{2} \lambda$ è costante $=6$, come facilmente si verifica; mentre nel caso dell'ellissoide, sia pure di piccolissimo schiacciamento, vi ha una linea (ellisse focale) in ogni punto della quale la detta funzione diviene infinita. Da ciò deriva che talune espressioni di $V_{i}$ che possono avere un significato fisico nel caso della sfera, non sono, neppure per approssimazione, usufruibili nel caso dell'ellissoide, anche poco differente dalla sfera; altre soluzioni adottabili sia per la sfera che per l'ellissoide, conducono, nei due casi, a distribuzioni molto differenti della densità in prossimità del centro. Vediamone un esempio.

Poniamo

$$
\Phi(\lambda)=\frac{1}{2 a^{2}}\left[\left(a^{2}+\lambda\right)^{2}-a^{4}\right]
$$

questa espressione soddisfà alla (36). Avremo

$$
\Delta_{2} \Phi(\lambda)=\frac{\Delta_{1} \lambda}{2}\left(\frac{1}{a^{2}+\lambda}+\frac{2}{b^{2}+\lambda}\right) \frac{a^{2}+\lambda}{a^{2}}+\frac{\Delta_{1} \lambda}{a^{2}} .
$$

Nel caso della sfera, chiamando $r$ il raggio vettore del punto $(x, y, z)$ Annali di Matematica, Sexie III, Tomo XVII. 
si ha $a^{2}+\lambda=r^{2}$, quindi $\Delta_{1} \lambda=4 r^{2}$. La $\Delta_{2} \Phi(\lambda)$, e con essa la densità $k_{1}$, tende dunque continuamente a zero coll'avvicinarsi del punto al centro. Nel caso dell'ellissoide di rotazione, invece, mentre $a^{2}+\lambda$ e $\Delta_{1} \lambda$ si annullano in tutti i punti interni all'ellisse focale, lungo questa linea si ha $a^{2}+\lambda=0$, $\Delta_{1} \lambda=4\left(b^{2}-a^{2}\right)$ e perciò $\Delta_{2} \Phi(\lambda)=\frac{6}{a^{2}}\left(b^{2}-a^{2}\right)$. La densità presenta quindi, nel piano equatoriale, una discontinuità, quando si attraversa la ellisse focale.

\$19. Altre espressioni della $V_{i}$. Ma possiamo alla $V_{i}$ attribuire altre espressioni, le quali, a differenza di quelle considerate nel $\$ 17$, non dànno luogo a discontinuità nei valori della densità.

Poniamo

$$
h=1-\frac{x^{2}}{a^{2}}-\frac{y^{2}+z^{2}}{b^{2}},
$$

e diamo a $V_{i}$ la espressione

$$
V_{i}=A+\frac{1}{D} f(h)
$$

dove

$$
D=\frac{x^{2}}{a^{4}}+\frac{y^{2}+z^{2}}{b^{4}}+B h, \quad B=\frac{\frac{1}{a^{4}}+\frac{z}{b^{4}}}{\frac{1}{a^{2}}+\frac{2}{b^{2}}} .
$$

La $A$.̀̀ una costante che ha lo stesso significato che nella (35). Assoggettiamo la $f(h)$ alle condizioni

$$
f(0)=0, \quad f^{\prime}(0)=\frac{\mu}{2 a b^{2}},
$$

e di più alle condizioni di essere finite e continue, insieme colla sua derivata prima, e di avere la derivata 2.a finita nell'intervallo $(0,1)$. Avremo

$$
\frac{\partial V_{i}}{\partial x}=-\frac{f^{\prime}(h)}{D} \frac{2 x}{a^{2}}-\frac{2 x f(h)}{a^{2} D^{2}}\left(\frac{1}{a^{2}}-B\right) \text {. }
$$

Alla superticie dell'ellissoide $E$

$$
\left(\frac{\partial V_{i}}{\partial x}\right)_{0}=\frac{-\mu x}{a^{3} b^{2} D}
$$


D'altra parte, pei punti della superficie stessa, la $D$.ha lo stesso valore che la $P$ che figura nelle formole del $\S 16$; sicchè ivi $\frac{\partial \lambda}{\partial x}=\frac{2 x}{a^{2} D}$; donde

$$
\left(\frac{\partial V_{1}}{\partial x}\right)_{0}=\frac{-\mu \cdot x}{a^{3} b^{2} D}=\left(\frac{\partial V_{i}}{\partial x}\right)_{0}
$$

Tenuto conto della espressione di $B$, si ha senza difficoltà

$$
\left.\begin{array}{c}
\Delta_{2} V_{i}=\frac{4 f^{\prime \prime}(h)}{D} S \frac{x^{2}}{a^{4}}-\frac{2 f^{\prime}(h)}{D} S \frac{1}{a^{2}}+\frac{4 f^{\prime}(h)}{D^{2}}\left(S \frac{x^{2}}{a^{6}}-B S \frac{x^{2}}{a^{4}}\right)+ \\
+\frac{8 f(h)}{D^{3}}\left(S \frac{x^{2}}{a^{8}}-2 B S \frac{x^{2}}{a^{6}}+B^{2} S \frac{x^{2}}{a^{4}}\right)
\end{array}\right\}
$$

dove il simbolo $S$ ha il significato indicato dalla

$$
S \frac{x^{2}}{a^{4}}=\frac{x^{2}}{a^{4}}+\frac{y^{2}+z^{2}}{b^{4}}
$$

ed analoghe.

Per le condizioni di regolarità cui la $f(h)$ si suppone soggetta, il 2. ${ }^{\circ}$ membro della (47), e con esso la densità $k_{1}$, rimangono finiti in ogni punto interno all'ellissoide $E$. Coll'avvicinarsi del punto $(x, y, z)$ al centro dell'ellissoide, lungo un qualsiasi raggio vettore, la $D$ tende con continuità al valore $B$ e la $\Delta_{2} V_{i}$ tenderà con continuità al valore $-\frac{2 f^{\prime}(1)}{B} S \frac{1}{a^{2}}$. Nè vi sarà differenza essenziale di comportamento della $\Delta_{2} V_{i}$ dal caso della sfera a quello dell'ellissoide; e si può notare che, col valore che abbiamo scelto per $B$, i termini dopo $\mathrm{i}$ due primi nel $2 .^{\circ}$ membro della (47) si manterranno sempre piccolissimi pel caso di un ellissoide poco differente dalla sfera.

Per avere un'idea del modo di variare di $\Delta_{2} V_{i}$ (e quindi della densità) nell'interno di $E$, vediamo che cosa diventa la (47) nel caso della sfera $(a=b)$. Allora, posto $x^{2}+y^{2}+z^{2}=r^{2}$,

$$
\begin{gathered}
B=\frac{1}{a^{2}}, \quad D=\frac{1}{a^{2}}, \quad h=1-\frac{r^{2}}{a^{2}} \\
\Delta_{2} V_{i}=4 f^{\prime \prime}(h) \frac{r^{2}}{a^{2}}-6 f^{\prime}(h) .
\end{gathered}
$$


Poniamo ad esempio:

$$
f(h)=A_{1} h+A_{2} h^{2}+A_{3} h^{3} ; \quad\left(A_{1}=\frac{\mu}{2 a b^{2}}\right)
$$

risulterà

$$
\Delta_{2} V_{i}=B_{1}+B_{2} \frac{r^{2}}{a^{2}}+B_{3} \frac{r^{4}}{a^{4}}
$$

dove

$$
\begin{gathered}
B_{1}=-6 A_{1}-12 A_{2}-18 A_{3}, \\
B_{2}=20 A_{2}+60 A_{3}, \quad B_{3}=-42 A_{3} .
\end{gathered}
$$

Le quantità $B_{1}, B_{2}, B_{3}$ sono legate alla sola condizione

$$
B_{1}+\frac{3}{5} B_{2}+\frac{3}{7} B_{3}=-6 A_{1}=\frac{-3 \mu_{4}}{a b^{2}} .
$$

$\S 20$. Le formole del paragrafo precedente stabiliscono in modo esatto quello che dalla teoria di Clarkaut era dimostrato soltanto in via approssimata, che cioè: distribuzioni di massa per strati omogenei, limitati da superficie di rotazione poco differenti da sfere, sono compatibili colla ipotesi che una superficie esterna di livello sia un ellissoide di rotazione schiacciato di piccola eccentricità. È facilissimo sviluppare in serie procedenti secondo le potenze della quantità $\frac{b^{2}-a^{2}}{a^{2}}=i^{2}$ il $2 .^{\circ}$ membro della (47). I termini indipendenti da $i^{2}$ coincidono naturalmente col $2 .^{\circ}$ membro della $\left(47^{\prime}\right)$. A meno di termini in $i^{4}$ si ha poi

$$
\begin{gathered}
h=1-\frac{r^{2}}{a^{2}}+\frac{i^{2}\left(y^{2}+z^{2}\right)}{a^{2}}, \quad B=\frac{1}{a^{2}}\left(1-\frac{2}{3} i^{2}\right), \\
D=\frac{1}{a^{2}}\left(1-\frac{2}{3} i^{2}\right)-\frac{i^{2}\left(y^{2}+z^{2}\right)}{a^{4}}+\frac{2}{3} \frac{i^{2} r^{2}}{a^{4}}, \\
S \frac{x^{2}}{a^{6}}-B S \frac{x^{2}}{a^{4}}=\frac{2}{3} \frac{i^{2} r^{2}}{a^{6}}-\frac{i^{2}\left(y^{2}+z^{2}\right)}{a^{6}} .
\end{gathered}
$$

L'ultimo termine, ossia la parentesi che moltiplica $\frac{8 f(h)}{D^{3}}$ nel $2 .^{\circ}$ membro della $\left(\begin{array}{l}4 \\ 4\end{array}\right)$, è dell'ordine di $i^{4}$. 
§ 21. Caso dell'ellissoide a tre assi. La ricerca di distribuzioni interne compatibili colla espressione della f.' potenziale esterna è ugualmente agevole nel caso dell'ellissoide a tre assi. Diamone un cenno.

Ho dimostrato, nella citata Memoria del 189 ( $\left.^{*}\right)$, che quando una delle superficie d'equilibrio esterne è un ellissoide a tre assi $(a, b, c)$ del quale l'asse $a$ coincida coll'asse di rotazione del pianeta, la f.' potenziale dell'attrazione sopra e fuori dell'ellissoide può scriversi

$$
V=\frac{1}{2} M V_{1}+k_{1} V_{2}+k_{2} V_{3}
$$

dove

$$
\begin{gathered}
V_{1}=\int_{\lambda}^{\infty} \frac{d s}{\sqrt{\bar{R}}}, \quad V_{2}=\int_{\lambda}^{\infty} \frac{1}{b^{2}+s}\left(1-\frac{x^{2}}{a^{2}+s}-\frac{3 y^{2}}{b^{2}+s}-\frac{z^{2}}{c^{2}+s}\right) \frac{d s}{\sqrt{\bar{R}}} \\
V_{3}=\int_{\lambda}^{\infty} \frac{1}{c^{2}+s}\left(1-\frac{x^{2}}{a^{2}+s}-\frac{y^{2}}{b^{2}+s}-\frac{3 z^{2}}{c^{2}+s}\right) \frac{d s}{\sqrt{\bar{R}}}
\end{gathered}
$$

$\lambda$ è la maggior radice dell'equazione

$$
\begin{gathered}
\frac{x^{2}}{a^{2}+\lambda}+\frac{y^{2}}{b^{2}+\lambda}+\frac{z^{2}}{c^{2}+\lambda}=1, \\
R=\left(a^{2}+s\right)\left(b^{2}+s\right)\left(c^{2}+s\right),
\end{gathered}
$$

$k_{1}$ e $k_{2}$ sono due costanti proporzionali al quadrato della velocità angolare e dipendenti da $a, b, c$. Posto

$$
\begin{array}{cc}
\frac{x^{2}}{a^{4}}+\frac{y^{2}}{b^{4}}+\frac{z^{2}}{c^{4}}=\frac{1}{D_{0}^{2}}, & B_{1}=\int_{0}^{\infty} \frac{d s}{\left(b^{2}+s\right)^{2} \sqrt{R}}, \\
A_{2}=\int_{0}^{\infty} \frac{d s}{\left(b^{2}+s\right)\left(c^{2}+s\right) \sqrt{R}}, & C_{1}=\int_{0}^{\infty} \frac{d s}{\left(c^{2}+s\right)^{2} \sqrt{R}}, \\
C_{2}=\int_{0}^{\infty} \frac{d s)\left(a^{2}+s\right) \sqrt{R}}{\left(c^{2}+s\right)\left(b^{2}+s\right) \sqrt{R}}, &
\end{array}
$$

(*) Rendiconti Lincei, III, $10^{\circ}$ sem., 1894, pag. 233. 
si deduce facilmente dalle (49) che nei punti della superficie dell'ellissoide

$$
\frac{\partial V_{1}}{\partial x}=\frac{2 x}{a^{3} b c} D_{0}^{2}
$$

ed analoghe :

$$
\begin{aligned}
& \frac{\partial V_{2}}{\partial x}=-2 C_{2} x+\frac{4 y^{2} x}{a^{8} b^{5} c} D_{0}^{2} \\
& \frac{\partial V_{2}}{\partial y}=-6 B_{1} y+\frac{4 y^{3}}{a b^{7} c} D_{0}^{2} \\
& \frac{\partial V_{8}}{\partial z}=-2 A_{2} z+\frac{4 y^{2} z}{a b^{5} c^{3}} D_{0}^{2}
\end{aligned}
$$

Si hanno analogamente le derivate della $V_{\mathrm{s}}$ cangiando nelle ultime tre formole $V_{2}$ in $V_{3}$ e permutando circolarmente le lettere $(x, y, z),(a, b, c)$.

Possiamo interpretare l'espressione (48) come la somma della f." potenziale di un ellissoide omogeneo e di quella di tre strati superficiali. Quanto al termine $\frac{M}{2} V_{1}$ esso è notoriamente la f.e p.e di uno strato distribuito sull'ellissoide $E$, la cui densità superficiale sia proporzionale alla distanza fra $E$ ed un ellissoide omotetico, concentrico, infinitamente prossimo. Quanto al termine $k_{1} V_{2}$, si osservi che posto

$$
\begin{gathered}
h_{s}=1-\frac{x^{2}}{a^{2}+s}-\frac{y^{2}}{b^{2}+s}-\frac{z^{2}}{c^{2}+s}, \\
U=\pi a b c \int_{2}^{\infty} \frac{h_{s} \cdot d s}{\sqrt{\bar{R}}},
\end{gathered}
$$

si può scrivere

$$
V_{2}=-\frac{1}{\pi a b^{2} c} \frac{\partial U}{\partial b}+\frac{1}{b^{2}} \int_{\lambda}^{\infty} \frac{h_{s} \cdot d s}{\sqrt{\bar{R}}} .
$$

Sicchè, salvo un fattore costante di proporzionalità, $V_{2}$ risulta la somma della $f:$ potenziale esterna di un ellissoide onogeneo, e di quella di uno strato superficiale disteso sull'ellissoide $E$ e di densità proporzionale alla distanza fra $E$ ed un ellissoide coassiale infinitamente prossimo, $i$ cui assi siano $a$, $b-\varepsilon, c$. $\dot{\mathrm{E}}$ facile verificare come una tale densità nel punto generico $(x, y, z)$ sia proporzionale a $y^{2}, D_{0}$. 
Similmente $V_{3}$ può interpretarsi come somma della f." potenziale esterna di un ellissoide omogeneo e di quello di uno strato superficiale compreso

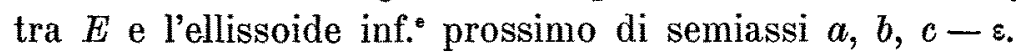

\$21. Si tratta ora di costruire delle funzioni $V_{1 i}, V_{2 i}, V_{3 i}$ le quali, all'interno dell'ellissoide $E$, siano finite insieme colle loro derivate 1.e, abbiano le derivate 2. finite, e siano tali che nei punti di $E$ si abbia:

$$
\begin{array}{cc}
V_{1 i}=V_{1}, \quad V_{2 i}=V_{2}, & V_{3 i}=V_{3}, \\
\frac{\partial V_{1 i}}{\partial r}=\frac{\partial V_{1}}{\partial r}, & \frac{\partial V_{2 i}}{\partial r}=\frac{\partial V_{2}}{\partial r},
\end{array}
$$

dove $r$ indica una qualunque delle coordinate.

Riguardo alla $V_{1 i}$ varranno gli stessi artificî che abbiam indicato per la costruzione della $V_{i}$ nel caso. dell'ellissoide di rotazione. Per la $V_{2 i}$ possiamo assumere

$$
V_{2 i}=\int_{0}^{\infty} \frac{d s}{\left(b^{2}+s\right) \sqrt{R}}-x^{2} C_{2}-3 y^{2} B_{1}-z^{2} A_{2}+\frac{2 y^{2}}{a b^{5} c} \Phi(\lambda),
$$

ove la $\Phi(\lambda)$ soddisfaccia alle condizioni

$$
\Phi(0)=0, \quad \Phi^{\prime}(0)=1,
$$

e di più $\Delta_{2} \Phi(\lambda)$ sia finita in ogni punto per valori di $\lambda$ fra 0 e $-a^{2}$. La $V_{2 i}$ si riduce evidentemente a $V_{2}$ per $\lambda=0$. Arremo poi

$$
\begin{aligned}
& \frac{\partial V_{2 i}}{\partial x}=-2 x C_{2}+\frac{2 y^{2}}{a b^{5} c} \Phi^{\prime}(\lambda) \frac{\partial \lambda}{\partial x} \\
& \frac{\partial V_{2 i}}{\partial y}=-6 y B_{1}+\frac{2 y^{2}}{a b^{5} c} \Phi^{\prime}(\lambda) \frac{\partial \lambda}{\partial y}+\frac{4 y}{a b^{5} c} \Phi(\lambda) \\
& \frac{\partial V_{2 i}}{\partial z}=-2 z A_{2}+\frac{2 y^{2}}{a b^{5} c} \Phi^{\prime}(\lambda) \frac{\partial \lambda}{\partial z} .
\end{aligned}
$$

Ricordando le espressioni delle derivate di $\lambda$, per $\lambda=0$, e tenendo conto delle (53) si verifica come queste derivate della $V_{2 i}$, sull'ellissoide $E$, risultino uguali a quelle della $V_{2}$ date dalle (50). 
Con calcoli facili $\left(^{*}\right)$ si ottiene poi

$$
\begin{gathered}
\Delta_{2} V_{2 i}=- \\
-\frac{4}{a b^{3} c}+\frac{2 y^{2}}{a b^{5} c}\left[\Phi^{\prime \prime}(\lambda) \cdot \Delta_{1} \lambda+\Phi^{\prime}(\lambda) \cdot \Delta_{2} \lambda\right]+ \\
+\frac{8 y}{a b^{5} c} \Phi^{\prime}(\lambda) \frac{\partial \lambda}{\partial y}+\frac{4}{a b^{5} c} \Phi(\lambda) .
\end{gathered}
$$

Questa espressione, divisa per $-4 \pi$, dà la densità spaziale di una massa la quale all'esterno di $E$ dà luogo alla funzione potenziale $V_{2}$. In simile modo si può costruire quella corrispondente al termine $V_{3}$ della funzione potenziale esterna.

$\left(^{*}\right)$ Occorre osservare che $\mathrm{i}$ tre integrali $A_{2}, B_{1}, C_{2}$ sono legati dalla relazione

$$
A_{2}+3 B_{1}+C_{2}=\frac{2}{a b^{3} c} \text {. }
$$

